\title{
An Unusual Karyotype in a Patient with Signs Suggestive of Down's Syndrome
}

\author{
W. J. PEPLER, MOYRA SMITH, and W. A. VAN NIEKERK
}

From the Departments of Physiology and Gynaecology of the University of Pretoria, Pretoria, South Africa

It is now generally accepted that Down's syndrome is due to the presence of extra genetic material of a chromosome in the G group. By convention, Down's syndrome is attributed to the presence of a triple dose of the genetic material of chromosome 21, but recent autoradiographic studies seem to suggest that the extra chromosome is in fact No. 22 (Patau, 1965). However, for the sake of convenience the extra chromosome in this paper will be referred to as No. 21 .

Patients with the stigmata of Down's syndrome are usually divided into two groups. The first group is comprised of those patients originally described by Lejeune, Gautier, and Turpin (1959) with a modal chromosome number of 47 and where the extra chromosome is indistinguishable from the autocentric autosomes in Group G. In the second group of patients only 46 chromosomes are present, the extra 21st chromosome being translocated onto another autosome. The translocation usually takes place onto another $G$ or to a $D$ chromosome. Another possibility in this group is the occurrence of an isochromosome of No. 21 .

The present paper describes a patient with features of Down's syndrome, with a modal number of 47 chromosomes and in whom the extra chromosome was found to be indistinguishable from the $F$ group.

\section{Case Report}

The patient, a white female aged 41 , has been an inmate of the Weskoppies Mental Institution in Pretoria for many years. No personal or family history could be obtained and as far as could be ascertained the patient was born in South West Africa.

On examination the skin was found to be dry and coarse and the hair straight and scanty. Her height was $140 \mathrm{~cm}$. (4 ft. $7 \mathrm{in}$.) and the cranial circumference measured $48 \mathrm{~cm}$. (19.25 in.). The face showed typical mongoloid features, with a small nose and chin, and the bridge of the nose was flattened (Fig. 1). The eyes were small and narrow but there was no sign of any upward slope; the eyebrows, however, did show an upward lateral slope. There was no epicanthic fold, but well- ir marked strabismus could be seen. Bilateral cataract formation was present and as a result the vision was poor. $\infty$ In addition, severe conjunctivitis was present at the time $ᄋ$ of examination. The mouth was very small, and the tongue did not appear to be enlarged, but showed deep $\vec{z}$ fissures. No arching of the palate could be seen. The ears were low-set and the pinnae much reduced in size. Hearing appeared to be normal. The neck was short and broad. The hands measured $13 \mathrm{~cm}$. (5 in.) in $\mathscr{8}$ length and $11.3 \mathrm{~cm}$. (4.5 in.) in breadth, and the thumb 9 was far from the second finger, but there was no obvious shortening or inward curve of the fifth finger. The feet were short and broad with gross bunion formation. Examination of the nervous system proved to be very difficult as it was not possible to make contact with the patient. She was unable to help herself, but her poor vision was probably a contributing factor. No abnormalities were detected on examination of the other systems.

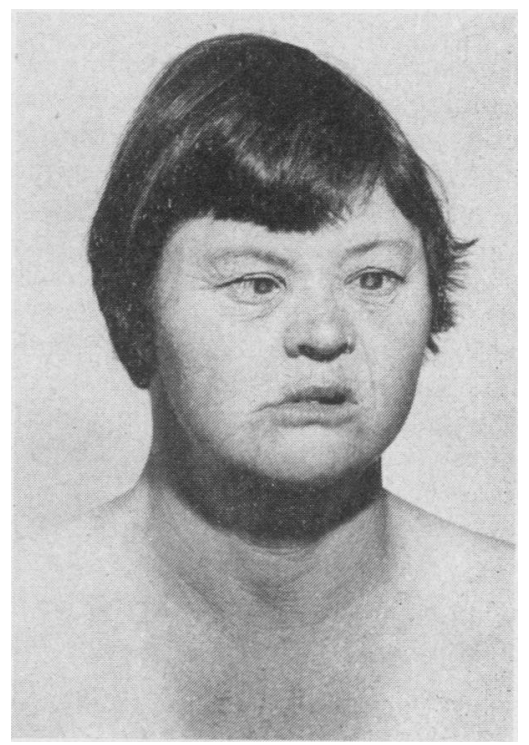

Fig. 1. Facies of patient. 

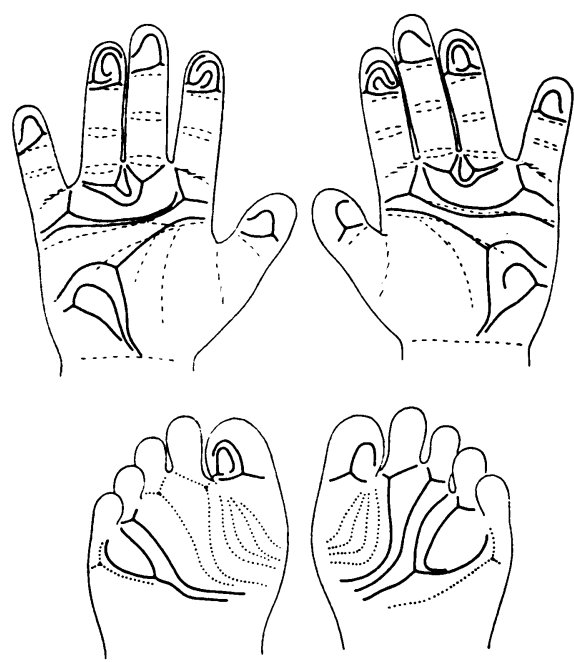

FIG. 2. Dermatoglyphic pattern of palm and sole prints.

Palm Prints. Palm and footprints were obtained and sent to Professor L. S. Penrose who reported that the dermal ridge patterns strongly indicated the diagnosis of mongolism. As can be seen from Fig. 2, the triradius $t$ is very near the centre of the palm, ulnar loops are present on the fingers, a digital loop is present between fingers III and IV, a thenar pattern is absent and on the feet no pattern can be seen on the hallucal area of the sole.

Special Investigations. Karyotyping was done by using both a direct bone-marrow preparation and also short-term peripheral blood cultures, according to a modification of the method described by Moorhead, Nowell, Mellman, Battips, and Hungerford (1960). The results are listed in the Table and a typical karyotype is shown in Fig. 3.

TABLE

NUMBER OF CHROMOSOMES IN BLOOD AND BONE-MARROW PREPARATIONS

\begin{tabular}{c|c|c|c|c}
\hline \multirow{2}{*}{ Tissue } & \multicolumn{4}{|c}{ Number of Chromosomes } \\
\cline { 2 - 5 } & $<45$ & 45 & 46 & 47 \\
\hline Bone-marrow & 4 & 3 & 4 & 41 \\
Blood culture & 4 & 5 & 4 & 61 \\
\hline
\end{tabular}

As can be scen from the karyotype, the extra chromosome in each of the cells containing 47 chromosomes was found to be a small metacentric chromosome indistinguishable from the two members of the 19th pair. The metaphase plates with 46 and less chromosomes show loss of different chromosomes on pairing.

Nuclear sex determination was done on buccal smears as well as on blood films. In the buccal smears $25 \%$ of

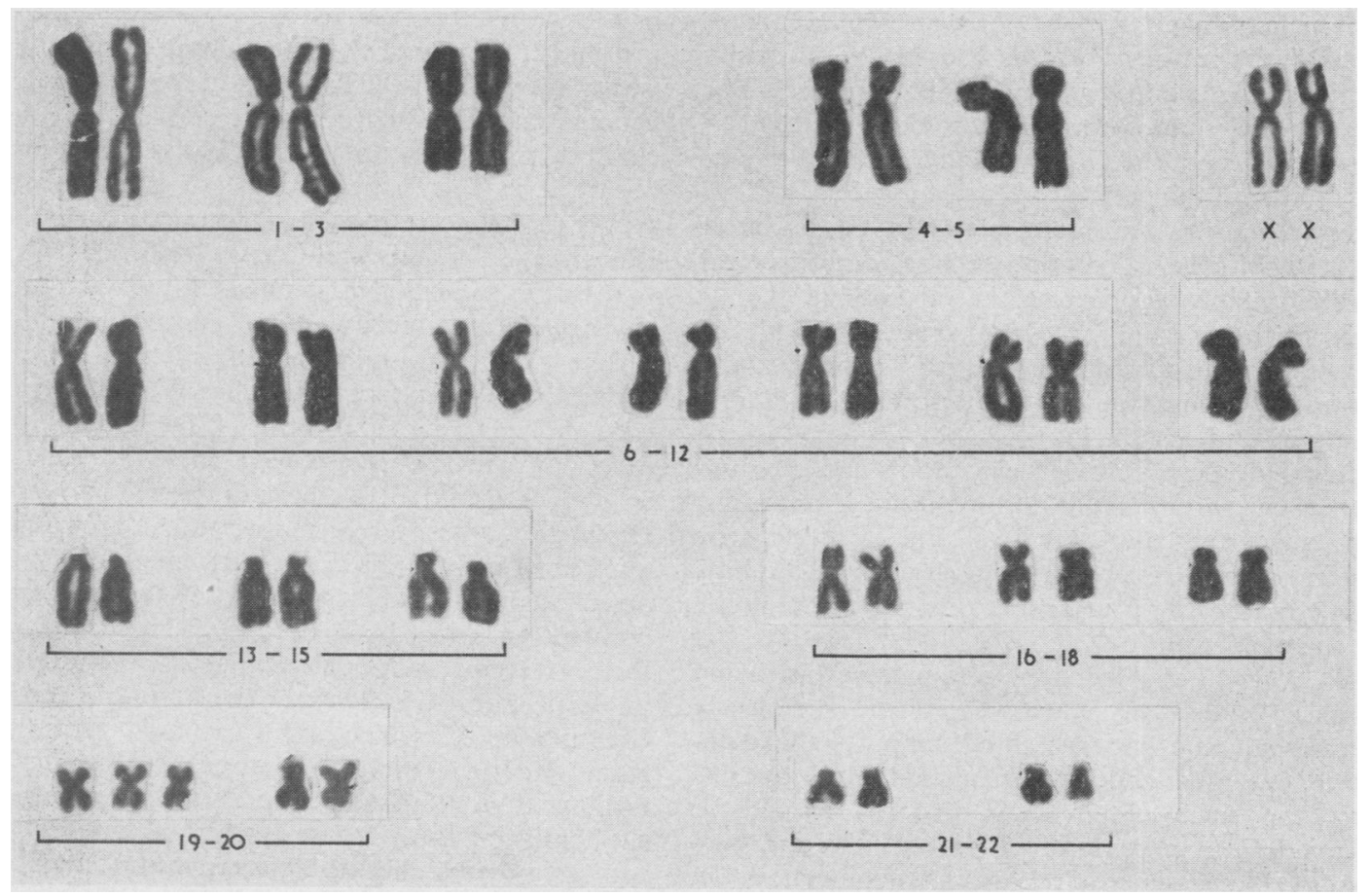

FIG. 3. Karyotype showing additional chromosome in group F. 
the cells were chromatin positive, and examination of 500 polymorphonuclear leucocytes revealed 6 drumsticks and 15 sessile nodules. The mean drumstick/nucleus ratio was 3.29. The method used for the determination of the drumstick and nuclear area was that described by Maclean (1962).

\section{Discussion}

Fraccaro, Kaijser, and Lindsten (1960) reported a family in which a mongol boy had a chromosome complement of 46 with only 3 chromosomes in group $\mathrm{G}$ and an additional chromosome in group $\mathrm{F}$. The father, however, was phenotypically normal and skin cultures showed a karyotype with 47 chromosomes with an additional chromosome in group $\mathrm{F}$ and 4 chromosomes in $\mathrm{G}$ group. It was suggested that the abnormality in the mongol boy was due to an isochromosome of the long arm of chromosome 21 and that the father was trisomic for chromosome 19. However, it is most likely that the 'trisomy-19' is a consequence of translocation in the father as well. Hamerton (1962) later reported that on analysis of peripheral blood cultures from the father, he was found to be mosaic, as cultures from the source only showed cells with 46 chromosomes and a normal male karyotype.

Hamerton, Briggs, Giannelli, and Carter (1961) also described a family in which blood cultures of the male mongol patient showed two cell lines namely, the majority of cells had 46 chromosomes with 3 group $\mathrm{G}$ and 5 group $\mathrm{F}$ chromosomes. The second cell line with 47 chromosomes had 4 chromosomes in the $\mathrm{G}$ group and 5 in the F group. The father of the patient also had a few cells with 47 chromosomes and an identical karyotype to that of his mongol son. The other cells were normal and no abnormality was detected in the mother.

A further case of Down's syndrome with 47 chromosomes and a karyotype with 5 group $\mathrm{F}$ and 3 group $\mathrm{G}$ chromosomes was described by Gray, Mutton, and Ashby in 1962: the maternal chromosome analysis revealed elongation and attenuation of the short arms of a $21 \mathrm{st}$ chromosome, and it was suggested that this was due to pericentric inversion of a 21 st chromosome, leading to duplication of part of the long arm of the 21st chromosome in the child.

In view of the fact that our patient has many clinical features of Down's syndrome and also a dermatoglyphic pattern which supports the diagnosis, one must conclude on phenotypical grounds that the extra chromosome material should be derived from chromosome No. 21. In order to explain the presence of the extra genetic material in the metacentric abnormal chromosome a few possibilities have to be considered. The first and $\Omega$

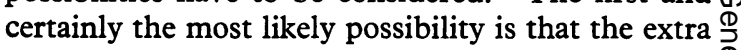
chromosome is an isochromosome for the long $\stackrel{\Phi}{\stackrel{\oplus}{+}}$ arms of No. 21, resulting from misdivision of the $\overrightarrow{\vec{A}}$ centromere during meiotic division of one of the $\frac{0}{7}$ parents or during mitotic division of the normal XX등 zygote with two chromosomes No. 21 . The latter $\frac{\bar{\sigma}}{\bar{\rho}}$ would require two events to explain the findings, $\frac{\sigma}{\sigma}$ the first one the formation of the isochromosome $\Omega$ and the second one a non-disjunctional process. $\mathrm{A} \%$ second hypothesis to consider is that the extra $\vec{O}$ chromosome is a pseudo-isochromosome resulting from the centric fusion of two No. 21 chromosomes. This would again necessitate not only the occurrence $\frac{S}{3}$ of the event which led to the interchange, but also a non-disjunctional event to explain the formation of $i r$ the tetrasomic cell line. However, the comple- $\vec{\sigma}$ mentary cell line to this should presumably be ${ }^{\infty}$ viable and therefore should have been found, so that this hypothesis seems unlikely. A third $\vec{z}$ possibility is that the extra chromosome is an interchange chromosome of the type $21 / 22$, resulting from reciprocal translocation of the centric fusion type between a No. 21 and a No. 22. A further possibility is of course that the extra chromosome is the result of an interchange between a chromosome No. 21 and another chromosome, which could be either a Y chromosome or an autosome. The last possibility is that the parent could have been a mosaic with a normal cell line and one containing an isochromosome for the long arm of No. 21. Segregation from the 47-chromosome cell line, as gonia, could then result in the formation of a karyotype as observed in this patient. The origin of such a secondary trisomic has been discussed by Polani, Hamerton, Giannelli, and Carter (1965).

In spite of the fact that no satellites were found on the abnormal chromosome, another possibility still has to be considered, and that is that the extra chromosome is in fact a member of the $G$ group with an enlarged short arm. Therkelsen (1964) described such chromosomes in a grandfather, mother, and child, the latter with Down's syndrome. Distinct satellites were demonstrable in only a small number of cells in his cases. Although elongation or enlargement of the short arm of the acrocentric chromosomes has been described in normal persons, it seems quite likely that the abnormality might produce an increased risk of non-disjunction. Seeing that this structural variant is transmitted from one generation to the next in Mendelian fashion, it would have been interesting to examine the family of the present patient. However, this was not possible, and therefore it cannot be determined with certainty whether the extra chromosome 
is in fact a $\mathrm{G}$ chromosome with enlarged short arms or whether it has arisen in the other ways suggested.

\section{Summary}

A 41-year-old white female patient, with the clinical and dermatoglyphic features of Down's syndrome, is described. Chromosome analysis of peripheral blood cultures and direct bone-marrow preparations revealed a modal number of 47 chromosomes in which the additional chromosome was found to be indistinguishable from the $F$ group. The possible derivations of this chromosome are discussed.

We are indebted to Professors L. S. Penrose and P. E. Polani for their advice.

\section{REFERENCES}

Fraccaro, M., Kaijser, K., and Lindsten, J. (1960). Chromosomal abnormalities in father and mongol child. Lancet, $1,724$.
Gray, J. E., Mutton, D. E., and Ashby, D. W. (1962). Pericentric inversion of chromosome 21. A possible further cytogenetic mechanism in mongolism. ibid., $1,21$.

Hamerton, J. L. (1962) (Ed.). Chromosomes in Medicine, pp. 156. (Little Club Clinics in Developmental Medicine No. 5). National Spastics Society and Heinemann Medical Books, London.

-, Briggs, S. M., Giannelli, F., and Carter, C. O. (1961). Chromosome studies in detection of parents with high risk of second child with Down's syndrome. Lancet, $2,788$.

Lejeune, J., Gautier, M., and Turpin, R. (1959). Étude des chromosomes somatiques de neuf enfants mongoliens. $C$. $R$. Acad. Sci. (Paris), 248, 1721.

Maclean, N. (1962). The drumsticks of polymorphonuclear leucocytes in sex-chromosome abnormalities. Lancet, $1,1154$.

Moorhead, P. S., Nowell, P. C., Mellman, W. J., Battips, D. M., and Hungerford, D. A. (1960). Chromosome preparations of leukocytes cultured from human peripheral blood. Exp. Cell Res., 20, 613.

Patau, K. (1965). Identification of chromosomes. In Human Chromosome Methodology, ed. J. J. Yunis, pp. 155-186. Academic Press, New York.

Polani, P. E., Hamerton, J. L., Giannelli, F., and Carter, C. O. (1965). Cytogenetics of Down's syndrome (mongolism). 3. Frequency of interchange of trisomics and mutation rate of chromosome interchanges. Cytogenetics, 4, 193.

Therkelsen, A. J. (1964). Enlarged short arm of a small acrocentric chromosome in grandfather, mother and child, the latter with Down's syndrome. ibid., 3, 441 . 